\title{
Structure and Function of Tripeptidyl Peptidase II, a Giant Cytosolic Protease
}

\author{
Anne-Marie Schönegge, Jürgen Peters, Marc Wehmer, Wolfgang Baumeister and Beate Rockel
}

Max-Planck Institute of Biochemistry, Molecular Structural Biology, Am Klopferspitz 18, D-82152 Martinsried, Germany

Tripeptidyl peptidase II (TPPII) is the largest known eukaryotic peptidase. It has been described as a 'multi-purpose peptidase', which, in addition to its house-keeping function in intracellular protein degradation, plays a role in several vital cellular processes such as cell division, apoptosis or antigen processing. Under conditions where the function of the proteasome is compromised, but also in certain diseases like muscle wasting and cancer, TPPII is upregulated. A number of studies have been performed to reveal its role in health and disease states, but although many functions have been ascribed to TPPII, its substrates or reaction partners have remained obscure [1-3].

TPPII has been classified as subtilase. However, only the N-terminal third of the peptide is homologous to subtilisin; the function of the larger part of the $140-150-\mathrm{kDa}$ polypeptide chain has remained enigmatic. In contrast to monomeric subtilisin, TPPII is a homooligomer with an unusual structure: Two strands composed of stacked dimers are wound into a large complex (5-6 MDa) with a spindle-like shape (Figure 1). In order to localize the different domains of the TPPII sequence in the holocomplex, we have studied TPPII complexes from Drosophila melanogaster (DmTPPII) and Homo sapiens (HsTPPII) by single-particle cryo electron microscopy and docking of high-resolution structures into the resulting 3D-maps $[4,5]$. Detailed analyses by maximum likelihood classification revealed that HsTPPII spindles are shorter than DmTPPII spindles and that their dimer-dimer and strand-strand contact areas are smaller. When not assembled, TPPII dimers have minimal activity, whereas full activity is only found in the assembled complex. According to our activation-model, which is based on the pseudoatomic structure of $D m$ TPPII-spindles, a loop connected to the active-site Serine must be rearranged in order to position this Serine in a catalytically-active position, and this rearrangement is triggered by the interaction of this loop with the neighbouring dimer in assembled TPPII-strands [4]. The comparison of the conformation of dimers at different positions in the complexes allowed us to pinpoint areas involved in this assembly-triggered activation and to monitor conformational differences occurring upon complex assembly. These areas include residues at the dimer-dimer interface and at the entrance of the large cavity system that harbors the active sites of TPPII [5].

TPPII can be found in most eukaryotic organisms. Interestingly, baker's yeast does not contain a TPPII homologue, whereas fission yeast does (SpTPPII). We have now set out to investigate $S p$ TPPII in terms of structure and enzymatic properties, since the comparison of structure and specificity of TPPII homologues from different organisms could provide further clues about the cellular function of TPPII and its reaction partners. 
References:

[1] B Tomkinson and AC Lindas, International Journal of Biochemistry \& Cell Biology 37 (2005), p. 1933.

[2] G Preta et al, Journal of Oncology Management 2010 (2010), p. 1.

[3] B Rockel et al, Biochimica Et Biophysica Acta-Proteins and Proteomics 1824 (2012), p. 237.

[4] CK Chuang et al, Nature Structural \& Molecular Biology 17 (2010), p. 990.

[5] A-M Schönegge et al, Structure 20 (2012), p. 593.

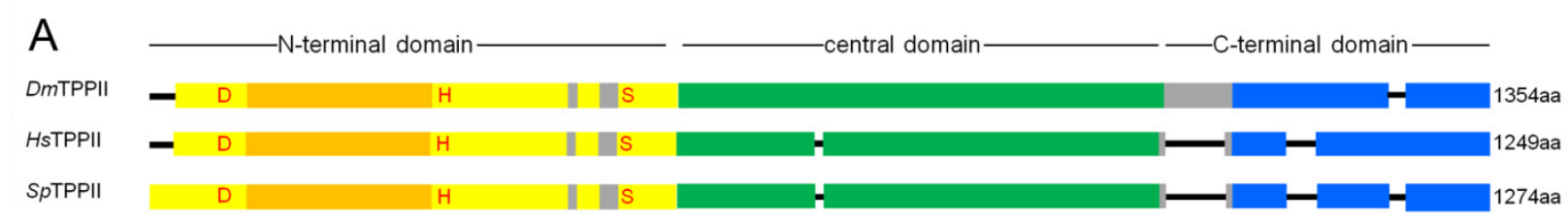

B

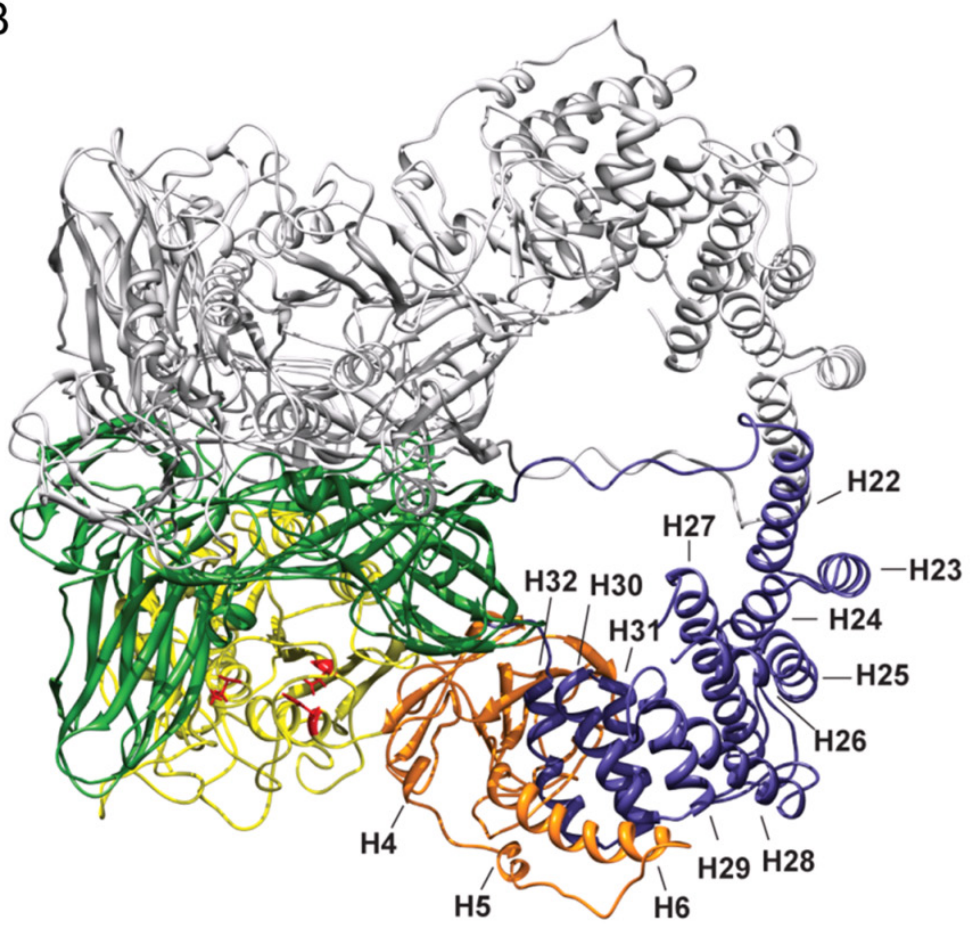

C

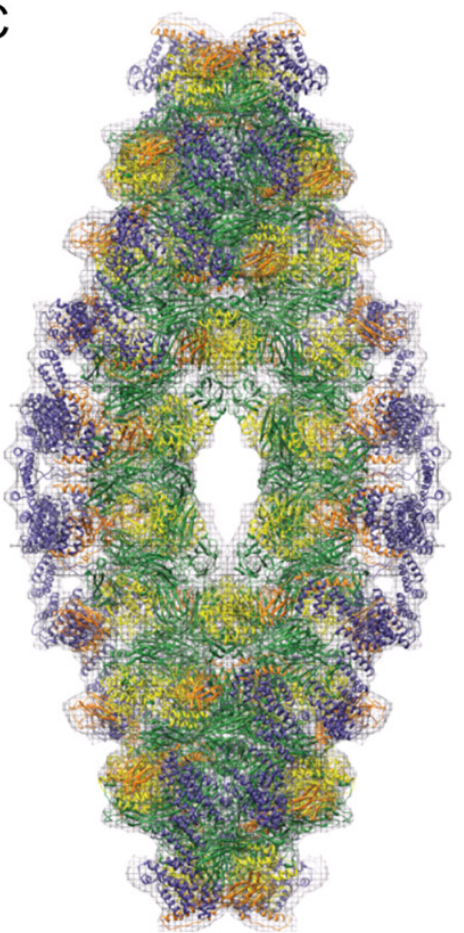

Figure 1. Hybrid structure of $H_{s}$ TPPII. A) Schematic sequence alignment of DmTPPII, $H_{s}$ TPPII and SpTPPII; yellow: N-terminal domain (subtilisin domain), orange: insert between the catalytic Asp and Ser residues, which is not present in subtilisin, red letters: residues of the catalytic triad; green: central domain, blue: C-terminal domain; gray: loops that are not resolved in the crystal structure of DmTPPII dimers. B) Ribbon diagram of a dimer of the comparative model of HsTPPII. The domains of one monomer are colored as in (A), the helices of the C-terminal domain are numbered. $\mathrm{C}$ ) Hybrid model of $H s$ TPPII; dimers are colored according to (A). 